\title{
Le commerce chinois à Dakar. Expressions spatiales de la mondialisation
}

Chinese trade in Dakar. Spatial expressions of globalisation

\section{Amadou Diop}

\section{OpenEdition}

1 Journals

\section{Édition électronique}

URL : http://journals.openedition.org/belgeo/7317

DOI : $10.4000 /$ belgeo.7317

ISSN : 2294-9135

Éditeur :

National Committee of Geography of Belgium, Société Royale Belge de Géographie

\section{Édition imprimée}

Date de publication : 31 décembre 2009

Pagination : 405-424

ISSN : 1377-2368

\section{Référence électronique}

Amadou Diop, «Le commerce chinois à Dakar. Expressions spatiales de la mondialisation», Belgeo [En ligne], 3-4 | 2009, mis en ligne le 17 mars 2013, consulté le 01 mai 2019. URL : http:// journals.openedition.org/belgeo/7317; DOI : 10.4000/belgeo.7317

Belgeo est mis à disposition selon les termes de la licence Creative Commons Attribution 4.0 International. 


\title{
Le commerce chinois à Dakar. Expressions spatiales de la mondialisation
}

\author{
Amadou Diop \\ Université Cheikh Anta Diop de Dakar
}

\begin{abstract}
RÉSUMÉ
L'irruption de la Chine dans le peloton des grandes puissances mondiales peut être considérée comme un des faits majeurs de ces 20 dernières années. Cette expansion chinoise a soulevé des enjeux spatiaux majeurs dans un contexte favorable de la mondialisation. Dans certains pays africains, la présence chinoise a débuté après les indépendances à travers de grands projets de développement. Actuellement, il s'agit d'une relance fondée sur de nouvelles bases avec des déplacements massifs de population via des canaux officiels ou non et des destinations diverses.

La destination du Sénégal a constitué pour les Chinois un cadre de sécurité, de facilité dans les importations des produits, mais surtout de profits commerciaux très bénéfiques. A Dakar, dans un espace aussi stratégique que le Boulevard Général De Gaulle communément appelé "Allées du Centenaire», les installations chinoises ont profondément modifié l'espace urbain. II s'est d'abord produit un important processus de «cantinisation" puis une recomposition sociale et spatiale très profonde. Acteurs principaux de ce dynamisme, les Chinois sont en collaboration avec les marchands ambulants, mais aussi avec la volonté des propriétaires des maisons qui profitent des transactions immobilières particulièrement lucratives.
\end{abstract}

MOTS-CLÉS: mondialisation, OPA Chinois, cantinisation, marchands ambulants, jeux des acteurs, gouvernance, Dakar

\begin{abstract}
CHINESE TRADE IN DAKAR. SPATIAL EXPRESSIONS OF GLOBALISATION

The emergence of China among the top world powers can be considered as one major fact in the last 20 years. China's expansion has raised crucial spatial stakes in the favourable context of globalisation. In some African countries, China's presence started after the independence with large-scale development projects. We currently witness a revival founded on news bases with massive population moves - official or not - towards various destinations. For the Chinese, Senegal represents a haven of security, easier imports of products, but most of all very high commercial profits. In Dakar, in the strategic Boulevard Général de Gaulle neighbourhood - commonly called "Allées du Centenaire»-, Chinese facilities have deeply modified the urban space. First of all, a considerable process of "canteenising" has taken place, followed by profound social and spatial reshaping. The Chinese, main players of this dynamism, collaborate with itinerant vendors, but also with homeowners who benefit from particularly lucrative real estate transactions.
\end{abstract}

KEY WORDS: globalisation, Chinese takeover bids, canteenising, itinerant vendors, players' strategies, governance, Dakar 


\section{INTRODUCTION}

$\mathrm{D}$ epuis 1978, date de lancement de la politique des «quatre modernisations»(1), par Deng Xiaoping, l'économie chinoise a connu d'importants progrès, devenant même l'une des plus performantes de la planète. Cette réussite qui s'explique en partie par l'arrivée massive des capitaux étrangers(2) dans le cadre de la promotion des zones économiques spéciales (ZES) est repérable à travers l'essor des exportations consécutif à la forte croissance de la production industrielle. La Chine est devenue le troisième exportateur mondial, derrière l'Allemagne et les Etats-Unis d'Amérique mais devant le Japon (Bénichi, 2006). L'Empire du Milieu enregistre un taux de croissance de 8 à $10 \%$ par an.

Le pari de la Chine est d'accumuler suffisamment de puissance pour contrôler et utiliser la mondialisation à son profit. C'est le sens de la fameuse formule de Deng Xiaoping selon laquelle «il faut ouvrir la fenêtre sans faire entrer les moustiques». Le maintien voire le renforcement de ses performances économiques passe par un approvisionnement abondant en matières premières et l'ouverture d'un marché extérieur pour l'écoulement des produits industriels. Ainsi, depuis quelques années, la Chine mène une grande offensive en Afrique.

Dans certains pays, la Chine a été présente, après les indépendances, dans les projets de développement ou dans l'appui aux mouvements dits de libération. Principalement localisés dans l'agriculture, la santé ou les infrastructures, ces projets ont nécessité, pour leur réalisation, le déplacement de populations encadrées par des conventions signées entre les pays. Aujourd'hui, avec la percée diplomatique de Pékin(3), la présence économique et commerciale chinoise est relancée sur de nouvelles bases et des déplacements de popula- tions via des canaux officiels ou non sont observés. II importe donc de s'intéresser aux modalités de l'accès des Chinois aux marchés du travail ainsi qu'aux réponses apportées par les gouvernements et les entrepreneurs africains. Pour le Sénégal, en particulier, la présence des opérateurs chinois modifie les règles de l'économie de bazar et les procédures de mise au travail au sein du secteur informel. Une partie de la main-d'oeuvre de ce secteur est devenue leur alliée. Une autre, notamment la frange commerçante supérieure, tente d'imposer des limites ou des freins à leurs activités en raison de la rude concurrence qu'ils imposent à certains entrepreneurs locaux. Cependant, les tentatives répétées des opérateurs consistant à pousser l'Etat à mettre un terme aux activités commerciales des Chinois ont eu peu d'effets. Et il n'a pas été noté, au Sénégal, l'exploitation de ce rejet à des fins politiques.

Les raisons du choix de la destination sénégalaise sont, entre autres, la sécurité, les facilités dans les importations des produits mais surtout des profits tirés du commerce. Le parcours migratoire des opérateurs chinois s'est construit sur des réseaux de proximité, le plus souvent familiaux, mais parfois professionnels. Pour la plupart des commerçants, I'homme est arrivé en premier et a mis en place la boutique, puis la femme est venue le rejoindre. Plus tard encore, si l'affaire tourne, un cousin viendra les seconder. Leur trajectoire est aussi marquée par des transits de courte durée dans certains pays comme la Côte d'Ivoire, le Mali et la Guinée(4).

L'irruption soudaine des Chinois à Dakar est en train de remodifier l'équilibre résultant du jeu des acteurs dans le processus du contrôle de l'espace urbain. Historiquement, à Dakar, comme dans la plupart des villes coloniales françaises, 
l'accaparement des instruments de domination, du contrôle de la centralité par les Européens et leurs intermédiaires libano-syriens s'est effectué progressivement en même temps que s'est amplifié le processus d'exclusion et d'infériorisation des autochtones. Après les indépendances, les Libano-Syriens prennent possession petit à petit des espaces stratégiques de la ville avec le retrait progressif des Blancs. Ces LibanoSyriens détenaient également le commerce en gros, une bonne partie de l'importation et le capital commercial. L'histoire y a construit, de nos jours, une autre réalité. Dans une période intermédiaire, qui s'étend jusqu'à une date récente, la conquête d'espaces commerciaux sénégalais a été l'emprise d'un groupe social assez spécifique constitué par les Moodu-moodu(5), qui ont fait preuve d'une bonne maîtrise de leur espace de déploiement économique, à travers l'extension du marché Sandaga(6) et la conquête des espaces appartenant jadis aux Libanais. La stratégie d'appropriation a consisté à enserrer les commerces de luxe libano-syriens dans les bazars appartenant à des Moodumoodu, concourant ainsi à déprécier l'espace. Ils ont ainsi progressivement réussi à supplanter les Libano-Syriens, obligés de s'éloigner petit à petit du secteur du commerce en détail et en gros pour s'orienter vers l'industrie et l'agrobusiness.

II faut noter que jusqu'aux années 2000, ce sont les commerçants sénégalais qui se rendaient en Chine pour s'approvisionner en marchandises. Conscients du potentiel de ce marché lointain, les Chinois ont décidé de s'installer à Dakar. Leur dynamisme et leurs techniques de vente efficaces leur ont permis de s'imposer rapidement.

La présence des opérateurs chinois modifie les règles de l'économie de bazar et les procédures de mise au travail au sein du secteur informel. Par le jeu d'alliances avec d'autres acteurs, les marchands ambulants, bénéficiant aux deux parties, ils sont en train de déstabiliser toutes les stratégies commerciales développées par les Moodu-moodu. Les commerçants chinois ont, en outre, besoin de ces relais locaux dont le dynamisme, la mobilité et la connaissance du terrain facilitent l'écoulement de leurs produits; quant aux marchands ambulants, ils y trouvent des moyens de subsistance dans un contexte économique qui ne leur est pas toujours favorable (difficultés d'accès au capital et au crédit pour mener des activités commerciales autonomes).

L'objectif de cet article est de suivre, d'identifier les mutations sociales, économiques et spatiales provoquées par le commerce chinois, d'analyser la logique du contrôle territorial des espaces centraux par le jeu des acteurs. II s'agit ensuite, dans une deuxième partie, de déterminer la manière dont ce commerce affecte la recomposition des territoires urbains, et favorise du coup l'émergence de nouvelles polarités, selon une dynamique d'appropriation/exclusion. Enfin, la contribution s'intéresse aussi aux impacts de cette activité sur l'économie locale, sur les relations entre commerçants chinois et acteurs locaux et sur l'environnement.

Le contexte géographique de l'étude porte sur le boulevard du général De Gaulle, réputé être le fief des Chinois à Dakar depuis quelques années, une sorte de «Chinatown sénégalais».

L'approche méthodologique a été basée sur une enquête auprès d'un échantillon représentatif de chefs de ménage du quartier des «Allées du Centenaire» et au niveau des clients fréquentant les échoppes chinoises. De plus, des informations qualitatives ont été recueillies grâce à des entretiens menés auprès des commerçants chinois, de leurs employés sénégalais et de marchands ambulants.

Ces enquêtes ont permis de disposer d'un vaste éventail d'informations sur l'occupation de l'espace, les dynamiques des mutations socio-spatiales et la perception qu'ont les populations dakaroises de cette migration chinoise. Une analyse thématique du contenu des entretiens a permis de mettre en évidence les tendances majeures des opinions des acteurs rencontrés sur les différentes problématiques de cet article. 


\section{L'OPA DES CHINOIS: PROCESSUS DE «CANTINISATION» DU QUAR- TIER DES ALLEES DU CENTENAIRE}

Dans la capitale sénégalaise, le choix des Chinois s'est porté sur le boulevard du Général de Gaulle (ou encore les Allées du Centenaire de Dakar(7)), devenu un grand bazar. Situé dans la partie sud de la ville de Dakar, ce boulevard bénéficie d'une localisation centrale. En effet, il constitue une porte d'entrée vers la zone portuaire et l'hypercentre, zone de concentration du haut tertiaire: banques, assurances, commerce et administration. Ce boulevard est en outre une courroie de transmission mettant en liaison le centre-ville, les principaux quartiers résidentiels de Grand-Dakar, des HLM(8) et des $\operatorname{SICAP}^{(9)}$. Enfin, il est situé à proximité des grands marchés(10) de Dakar qui l'enserrent et auxquels il est relié par d'importants axes transactionnels.

Le boulevard du Général de Gaulle a bénéficié jusqu'ici d'un habitat de type planifié avec un standing appréciable. II abritait une classe moyenne composée de hauts fonctionnaires de l'administration publique et des salariés du privé dont les revenus permettaient de préserver un cadre de vie acceptable. Ce boulevard est d'ailleurs comparé par certains Sénégalais aux «Champs Elysées» à Paris. Place symbolique, c'est là qu'est organisée le 4 avril de chaque année, la fête commémorative de l'indépendance du Sénégal qui consacre son accession à la souveraineté internationale.

En plus de la localisation stratégique dont il bénéficie, le choix du boulevard du Général de Gaulle a été déterminé par le coût élevé des loyers dans les ruelles commerçantes du centre-ville, et par la difficulté d'y trouver des surfaces disponibles. Cette large avenue ombragée, bordée de trottoirs et de pistes cyclables similaires à celles des grandes villes chinoises, permet aux activités commerciales de déborder sur la chaussée sans gêner le trafic. Ce lieu, bénéficiant d'une proximité du centre des affaires de Dakar, offre une très grande visibilité aux activités des com- merçants chinois qui ont d'ailleurs développé des stratégies pour s'en emparer, avec notamment l'achat de certaines maisons environnantes, en vue de mieux établir leur commerce.

Parmi les premiers occupants des Allées du Centenaire, certains sont partis en retraite et d'autres sont décédés. Avec l'agrandissement de la taille des ménages et la perte de revenus, une partie des nouveaux héritiers ne parvient plus à maintenir le même standing de vie. C'est pourquoi on observe de nouvelles stratégies d'occupation de l'espace. Elles se caractérisent par l'ouverture ou la transformation des garages des maisons et des espaces non bâtis qui bordent les allées en ateliers de couture, salons de coiffure, restaurants ou boutiques. En effet, dans ce contexte de crise, la manne financière du loyer chinois apparaît comme une bouffée d'oxygène: «A partir de 2002, les Chinois n'ont pas eu trop de peine à convaincre les propriétaires. Car le coût de la vie a bien augmenté dans la capitale sous l'effet de la dévaluation du franc CFA et du conflit ivoirien, qui a déplacé vers le Sénégal la plupart des grosses entreprises et des organisations internationales d'Abidjan. La manne financière des loyers chinois était la bienvenue» (Michel et Beuret, 2008).

Le processus de "Cantinisation»(11) a entraîné une modification des devantures des maisons avec la construction de cantines dont la mise en location a constitué une importante source de revenus pour ces populations riveraines. Les premiers à mettre leurs maisons en location et à construire des cantines l'ont fait pour des raisons économiques. Cependant, une deuxième vague a fini par «céder à la pression» et a mis en location soit la maison, soit les cantines, suite à la transformation du quartier en marché avec ses corollaires: pollution sonore, insalubrité, insécurité, problèmes d'accessibilité. Cependant, certains habitants refusent d'abandonner leurs 


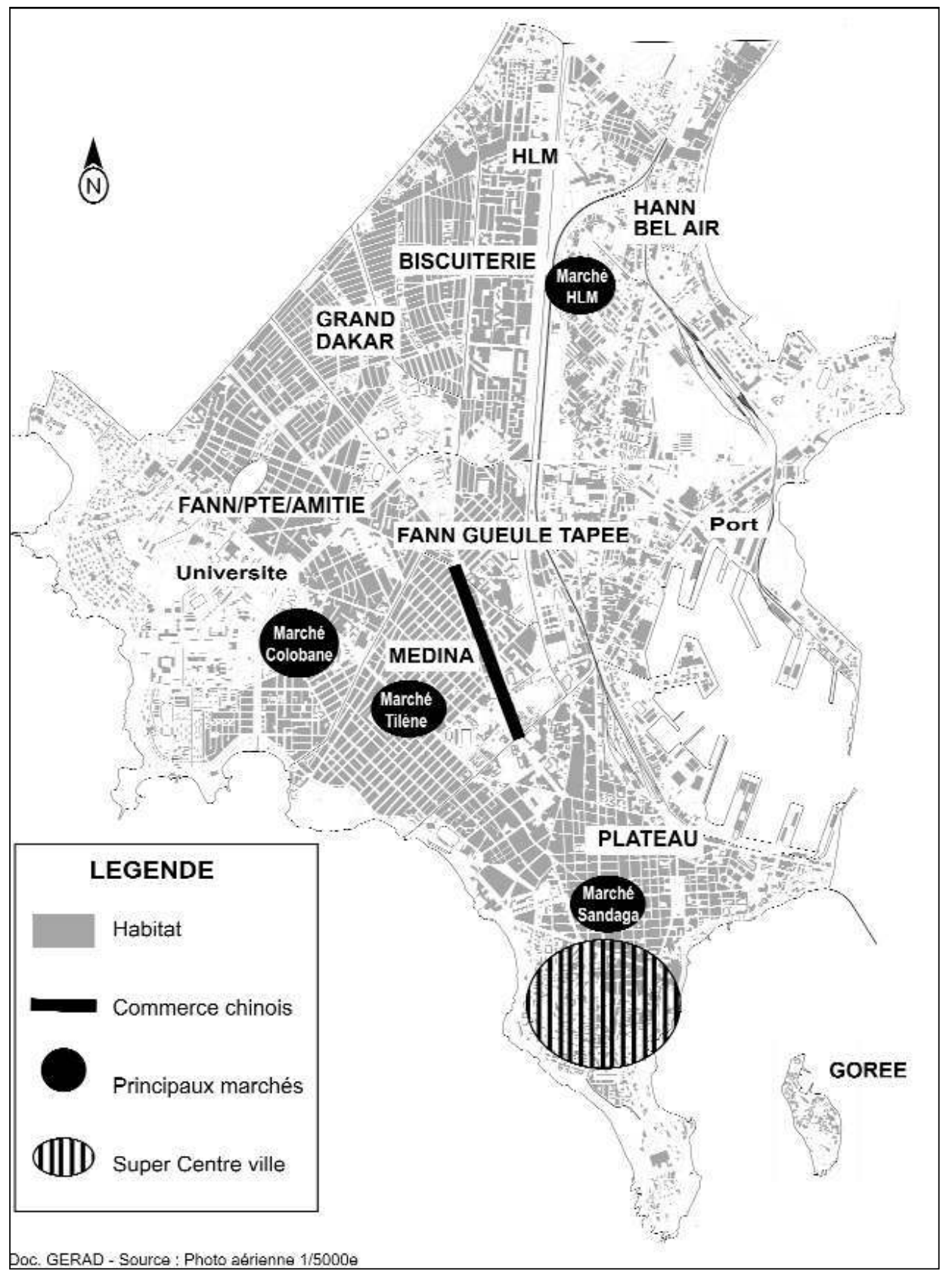

Figure 1. Localisation stratégique des allées du Boulevard Général de Gaulle. 
maisons. Dans certains cas, un membre de la famille a un emploi, ce qui permet de conserver le standing de vie d'antan, tandis que d'autres survivent en profitant de la présence des Chinois pour faire du petit commerce ou en devenant des employés de commerce de ces Chinois. Aussi sont-ils nombreux, les jeunes du quartier, à assister les commerçants chinois dans la vente des produits.

Les figures 2 et 3 , relatives aux éléments d'occupation de l'espace, montrent le processus de modification du tissu résidentiel.

Jusqu'en 1990, le quartier semblait encore garder une trame urbaine aérée marquée par un agencement en damier des différents ilots, même si les espaces verts et autres lieux publics subissaient, sous l'effet du temps combiné au manque d'entretien, une dégradation marquée. L'occupation de l'espace faisait état d'une certaine harmonie des dispositifs d'urbanisme. Les types d'habitations sont des maisons à deux étages bordées régulièrement, au niveau des intersections, par des immeubles. Les premiers magasins étaient aménagés au bas des immeubles. Ils étaient attribués en location vente d'un coût mensuel de 15000 fcfa ( 1 euro $=655,99$ Fcfa), pour une durée de 15 ans. Les principaux équipements dénombrés, bénéficiant d'une localisation stratégique au niveau des carrefours (au total 34) étaient constitués principalement de commerces de détail et de services divers (banques, pharmacies, etc.).

La détermination de la date d'implantation des équipements commerciaux permet de fournir une première appréciation de la "conquête» de ce quartier par les Chinois et illustre la diffusion spatiale des activités de commerce.

Sur 147 magasins de commerce répertoriés de nos jours le long du boulevard, 125 sont occupés par des commerçants chinois, dont le rythme d'installation a suivi ces dates clés:

- I'implantation débute en 2001-2002 avec l'installation de 10 Chinois. Venus des campagnes chinoises, ils sont pour la plupart âgés de 30 à 50 ans. Pendant leur trajectoire migratoire ils ont séjourné dans certaines villes chinoises (Beijing, Henan, Fujian) avant de venir à Dakar;

- entre 2003-2004 on peut parler d'un véritable boom de création de magasins avec une installation de 63 Chinois, soit $50 \%$ des implantations totales;

- les installations effectuées entre 20052006 sont de 37 magasins;

- entre 2007-2008, avec l'effet de saturation, le nombre d'installations est tombé à 15 magasins.

Les prix de location des magasins constituent pour certaines familles une importante source de revenus. Le coût du loyer est compris entre 150000 Fcfa et 300000 Fcfa. Aujourd'hui, la valeur locative d'une cantine est deux à trois fois supérieure au coût du loyer de la maison en 1990 et qui était estimé entre 40000 Fcfa et 60000 cfa. Par rapport au prix de location des magasins, qui était de 15000 Fcfa, la valeur locative d'aujourd'hui lui est dix à vingt fois supérieure.

Le statut d'occupation a ainsi fortement évolué suite à l'appauvrissement progressif des familles et des offres faites par les Chinois. Ces derniers accaparent progressivement les logements: les locataires consentent une importante avance qui permet au logeur de construire d'autres pièces en hauteur, afin de remplacer celles cédées aux locataires; ensuite des prêts sont consentis aux chefs de ménages souvent dans le besoin. Quand ces derniers se retrouvent, plus tard, dans l'impossibilité de rembourser, les Chinois les acculent et leur proposent l'achat des maisons. Ainsi, la différence entre le prix retenu et la valeur de la dette contractée est vite calculée et les lieux changent de propriétaire. Cette pratique fréquente a permis à des commerçants chinois d'être propriétaires de locaux qu'ils occupent, même si $30,3 \%$ résistent encore à la tentation de céder leurs maisons.

Les Chinois ont fait une Offre publique d'achat (OPA) sur les maisons de ce boulevard. Dans ces maisons entièrement louées, des quantités importantes de marchandises sont stockées dans les chambres, les transformant ainsi en de gigantesques dépôts de marchandises. La forte implantation des commerçants 


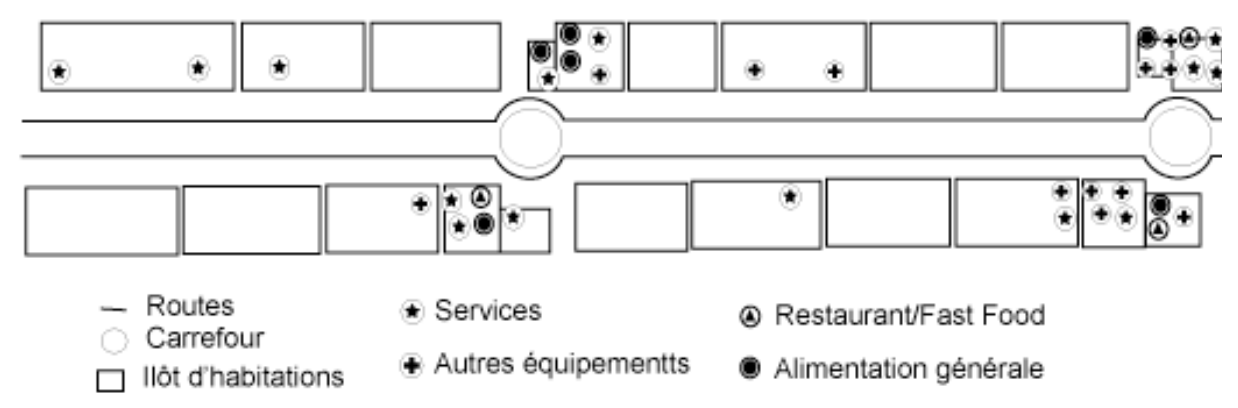

Figure 2. Occupation de l'espace en 1990.

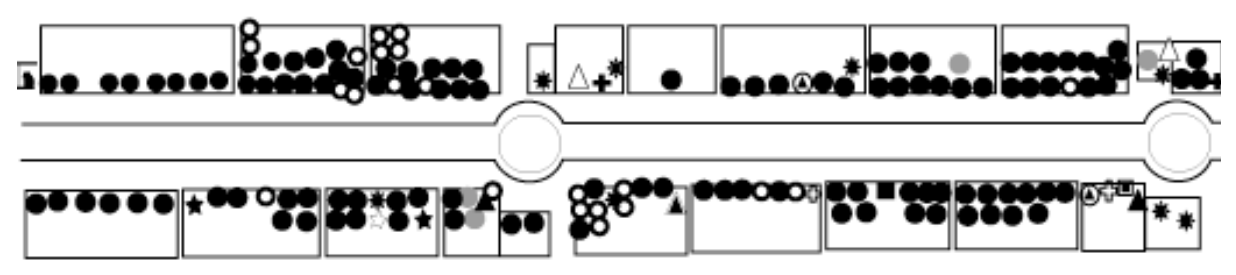

- Routes

- Carrefour

$\square$ llôt d'habitations

\section{Commerces}

- Commerce Chinois

O Commerce Sénégalais

Commerce autre Nationalité

\section{Services}

T. Station d'essence

+ Pharmacie

$\Delta$ Agence de voyage

Prescolaire

$\triangle$ Banque
- Auberge

(4) Restaurant

๑ Boulangerie

Source : Enquetes terrain, 2008

Figure 3. Occupation de l'espace en 2008: OPA chinoise.

chinois sur les Allées du Centenaire en particulier a entraîné d'importantes mutations sociales et spatiales se traduisant par l'émergence d'une nouvelle polarité et la constitution d'une classe marchan- de constituée par les commerçants chinois et leurs employés sénégalais. Ce phénomène s'inscrit dans un processus plus global de recomposition sociale et spatiale observé à Dakar. 


\section{LA RECOMPOSITION SOCIALE ET SPATIALE}

Noeud privilégié de communication nationale et internationale, Dakar est une métropole. Pendant la période coloniale, l'implantation des Européens a conditionné la structure actuelle du centre et les traits liés à son développement ont engendré une structure spatiale ségrégative. La zone de domination par excellence est le centre dont les instruments spatiaux ont été mis en place par le système colonial. La construction de la centralité s'est opérée à partir du port dont les caractéristiques du site offraient des facilitations commerciales. Celle-ci s'est forgée progressivement à partir de la superposition de plusieurs pouvoirs politiques, économiques et socioculturels se renforçant mutuellement et résultant de la combinaison de plusieurs variables significatives: infrastructures de base, équipements politiques, administratifs et commerciaux.

La morphologie urbaine actuelle se développe selon de nouvelles logiques d'éclatement urbain dans la région dakaroise. Le rythme effréné de la croissance à Dakar se manifeste, en effet, par l'étalement urbain et l'apparition de nouvelles polarités. L'évolution de la centralité dakaroise conduit à la genèse d'une polycentralité.

La tendance à la multipolarité s'est matérialisée par l'émergence d'un pôle constitué par l'espace du triangle Sud (place Sfax) avec les nouveaux locaux de la Radio Télévision Sénégalaise (RTS), la Banque de l'Habitat du Sénégal (BHS), l'agence nationale de la BCEAO, le Centre Africain d'Etudes Supérieures en Gestion (CESAG), l'aménagement du boulevard du Général de Gaulle, et l'implantation d'un centre commercial (les $4 \mathrm{C}$ ).

Une autre centralité est apparue, depuis plusieurs années, avec la mise en place du domaine industriel de la SODIDA pour délocaliser certaines usines du port de Dakar et encourager la création de petites unités industrielles sur des sites plus appropriés. Cela a engendré l'implantation de petites et moyennes industries et entreprises près des zones rési- dentielles. Le troisième pôle qui s'observe actuellement réside dans le déplacement des activités de services vers des zones résidentielles de la banlieue ou proches d'elle (VDN, Ngor, Almadies, Yoff, Nord Foire).

Dans le même ordre d'idées, les aménagements identifiés au niveau du boulevard Général de Gaulle, communément appelé «Allées du Centenaire de Dakar», ont fini par créer une nouvelle centralité linéaire avec l'installation des commerçants chinois sur ces deux façades. Cette dernière est la conséquence du dynamisme économique et démographique que connaît ce quartier. Le développement progressif d'espaces commerciaux en d'autres lieux s'explique par la difficulté de leur acquisition en ville. De plus en plus, on assiste à un renchérissement des locaux commerciaux dû à la rareté des terrains et emplacements disponibles. Avec la pression des besoins chinois immédiats, le quartier du Centenaire a connu un développement fulgurant au cours de cette quinquennale, impulsé par la crise de l'emploi. Face à cette situation, le secteur informel apparaît comme une réponse à l'incapacité du secteur moderne à offrir une activité accessible et suffisante, et présente une importante capacité de résorption du chômage.

Le quartier du Centenaire est devenu, à la faveur du commerce chinois, un important pôle commercial. En effet, cet espace semble fonctionner dans le cadre d'échanges et de rapports de complémentarité et de dépendance en chaîne qui s'instaurent entre les marchés, la zone portuaire, la zone ferroviaire, les banques et l'administration. On note des échanges intenses entre les marchés qui enserrent le boulevard et qui deviennent de plus en plus des lieux d'écoulement des produits chinois. Les échanges se font entre les banques, les zones portuaire et ferroviaire, et constituent par leur quantité un ensemble fonctionnel très lié.

L'aire d'influence des produits vendus à 
partir de la zone de provenance des clients montre qu'elle dépasse largement les limites du territoire national. Ces clients étrangers viennent particulièrement des pays limitrophes, principalement le Mali, la Guinée Conakry, la
Guinée Bissau, ou encore la Sierra Léone. Cette clientèle hétérogène, composée surtout de femmes, relève de toutes les couches sociales. Les produits proposés sont à la portée de toutes les bourses.

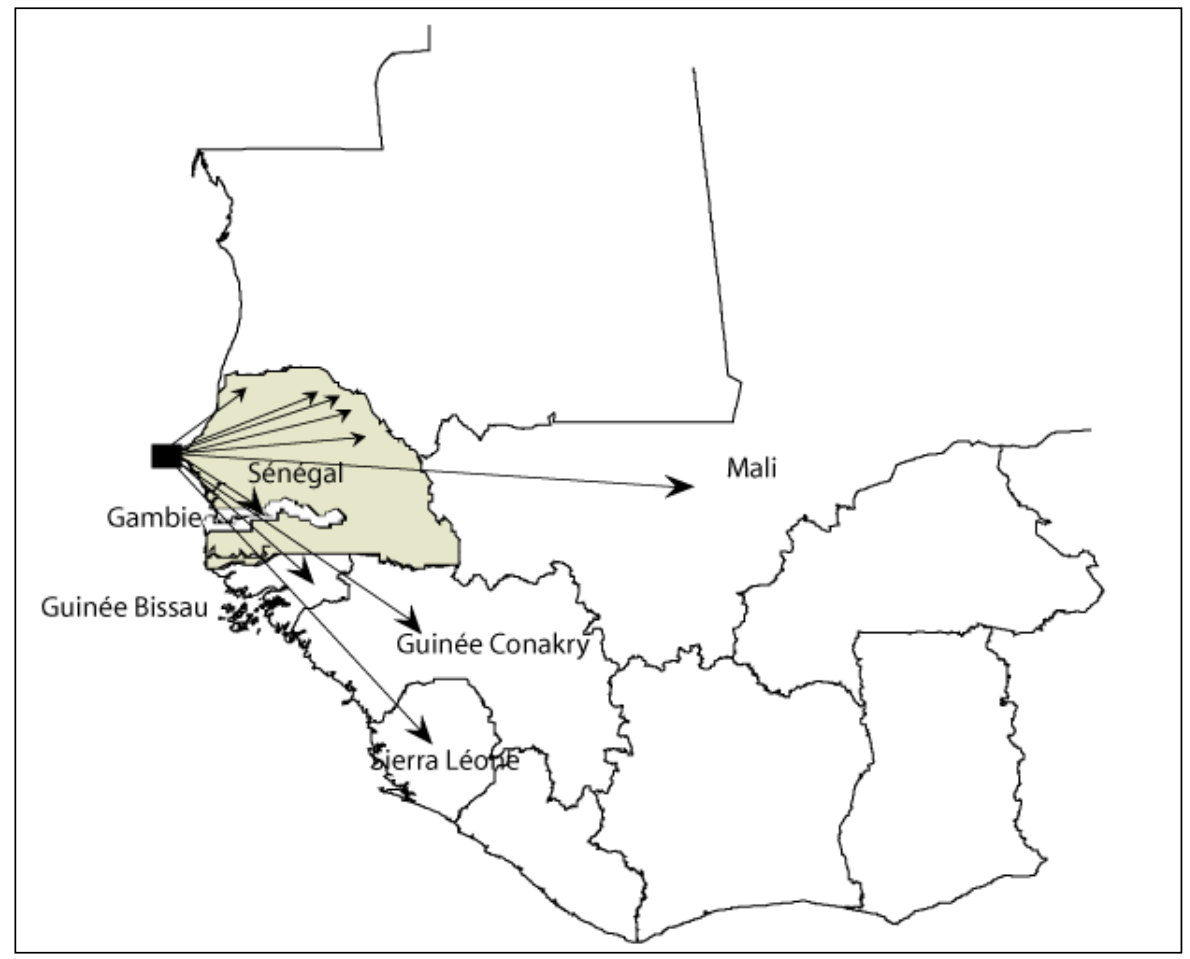

Figure 4. Aire de polarisation du commerce chinois.

\section{LE RENFORCEMENT DE L'ÉCONOMIE INFORMELLE, SOURCE D'EM- PLOI DES JEUNES}

La présence des marchands ambulants sur les Allées du Centenaire est concomitante à l'irruption soudaine des commerçants chinois. Ces marchands proviennent aussi bien des quartiers de Dakar que de l'intérieur du pays. Les commerçants chinois leur vendent en gros ou demi-gros leurs marchandises qu'ils revendent sur place, dans les quar- tiers, dans les autres marchés ou dans les régions.

L'arrivée des commerçants chinois à Dakar a créé une nouvelle économie informelle. Les devantures des boutiques sont devenues les lieux d'un exercice de survie quotidienne. La force de la petite production marchande (M. Santos, 1975) tient à sa souplesse et son ubiquité: la 
floraison des marchands ambulants en est une parfaite illustration.

Les Chinois développent également une stratégie de fidélisation de la clientèle en offrant des possibilités de paiements différés ou de reprise des produits invendus. Les recettes journalières des marchands ambulants sont comprises entre 15000 et 60000 F CFA, contre 100000 et $300000 \mathrm{~F}$ CFA pendant les périodes de fête. "Nous suivrons les Chinois partout et nous ne partirons jamais tant qu'ils sont là» affirmait un marchand ambulant lors de nos enquêtes. L'arrivée des commerçants chinois a permis à une tranche de la population de trouver du travail, ce qui représente une bouffée d'oxygène pour des jeunes, contraints jusqu'ici au désoeuvrement.

Ces activités dites informelles sont susceptibles de garantir la subsistance d'une sous-population urbaine caractérisée par l'incertitude de ses ressources économiques, même si la sécurité dans l'emploi et le revenu ne sont pas assurés. L'argument de la création d'emplois semble l'emporter sur la détermination des riverains à lutter contre l'occupation anarchique de leur environnement immédiat.

Les montants dépensés par les marchands ambulants varient de 10000 à $1000000 \mathrm{~F}$ CFA selon les périodes (jours ordinaires, fêtes). «Avant l'arrivée des Chinois, nous nous approvisionnions auprès des commerçants du marché Sandaga. Aujourd'hui, les commerçants chinois sont nos premiers fournisseurs» affirme un marchand ambulant. La revente des produits chinois leur permet de faire des marges financières importantes (du tiers au double) avec une fréquence d'écoulement rapide des marchandises grâce aux clients venus de divers quartiers de Dakar et des régions de l'intérieur du Sénégal, mais aussi grâce aux prix de vente fortement réduits. En plus des marchands ambulants, les Chinois sont devenus les principaux fournisseurs de plusieurs commerçants sénégalais. En effet, les grands commerçants de Sandaga comme ceux des HLM ciblent les magasins chinois pour l'achat en gros. Même les Libanais sont devenus les clients des Chinois et sont particulièrement intéressés par les chaussures qu'ils achètent en grande quantité.

L'aire de polarisation du commerce chinois s'étend à la sous-région. Des clients venus de la Guinée-Conakry, du Mali, de la Gambie, de la Guinée-Bissau, de la Sierra Léone se ravitaillent mensuellement chez les Chinois du boulevard Centenaire de Dakar. Les grands commerçants portent leur choix sur des produits d'esthétique rares en grande quantité qu'ils stockent pour être revendus en gros ou au détail selon la demande.

A ces clients revendeurs s'ajoutent les marchands d'étals qui occupent les devantures des magasins chinois. Bien qu'étant source d'encombrement, ces occupations sont favorisées par les Chinois qui approvisionnent ces marchands. Ces derniers en compensation sont aussi chargés de veiller sur les marchandises des Chinois.

Près de 160 jeunes sont employés par les Chinois avec des revenus mensuels qui varient entre 40000 et $75000 \mathrm{~F}$ CFA, à l'image de cet homme, originaire de la région du fleuve Sénégal et qui travaille dans une cantine chinoise depuis deux ans. II perçoit 60000 F CFA par mois et, avec ce travail, il envoie mensuellement 25000 F CFA à ses parents. II précise que c'est son premier travail. S'il déplore l'occupation anarchique du boulevard du Centenaire, il apprécie néanmoins la présence chinoise qui lui a permis de trouver du travail. Ces employés sans contrat facilitent la communication avec les clients et jouent le rôle d'intermédiaires entre ces derniers et leurs employeurs chinois. La présence de ces Sénégalais est donc indispensable à l'intégration des Chinois et au bon fonctionnement de leurs activités. Dans le magasin, le Chinois est en position d'arrière-plan et laisse l'employé en contact direct avec les clients. L'argent de la vente est aussitôt remis à l'employeur.

Cependant, des conflits entre employés et employeurs sont assez fréquents. Tout d'abord, plusieurs employés se plaignent de la longueur des journées de travail. Un employé de Chinois nous confie qu'un de leurs camarades a été licencié 
Ce client est venu de Fatick (à 155 kilomètres de Dakar, vers le centre), pour s'approvisionner en vêtements. Interrogé sur sa petite randonnée dans les dédales du "Chinatown" du Centenaire, il explique que cette visite va lui permettre de remplir son sac en jeans et autres "bodys" pour jeunes filles. "C'est un ami commerçant qui m'a soufflé l'idée, ajoute t-il. C'est pourquoi, je viens m'approvisionner ici. Arrivé à Fatick, je pourrai écouler la marchandise avec une bonne marge bénéficiaire ».

Bonnet sur la tête, ce commerçant, est venu de Kaffrine (nouvelle région, dans la zone centre du Sénégal, à 256 kilomètres de Dakar) pour les mêmes raisons : "les marchandises chinoises coûtent moins cher, ce qui nous permet de faire des marges bénéficiaires importantes ».
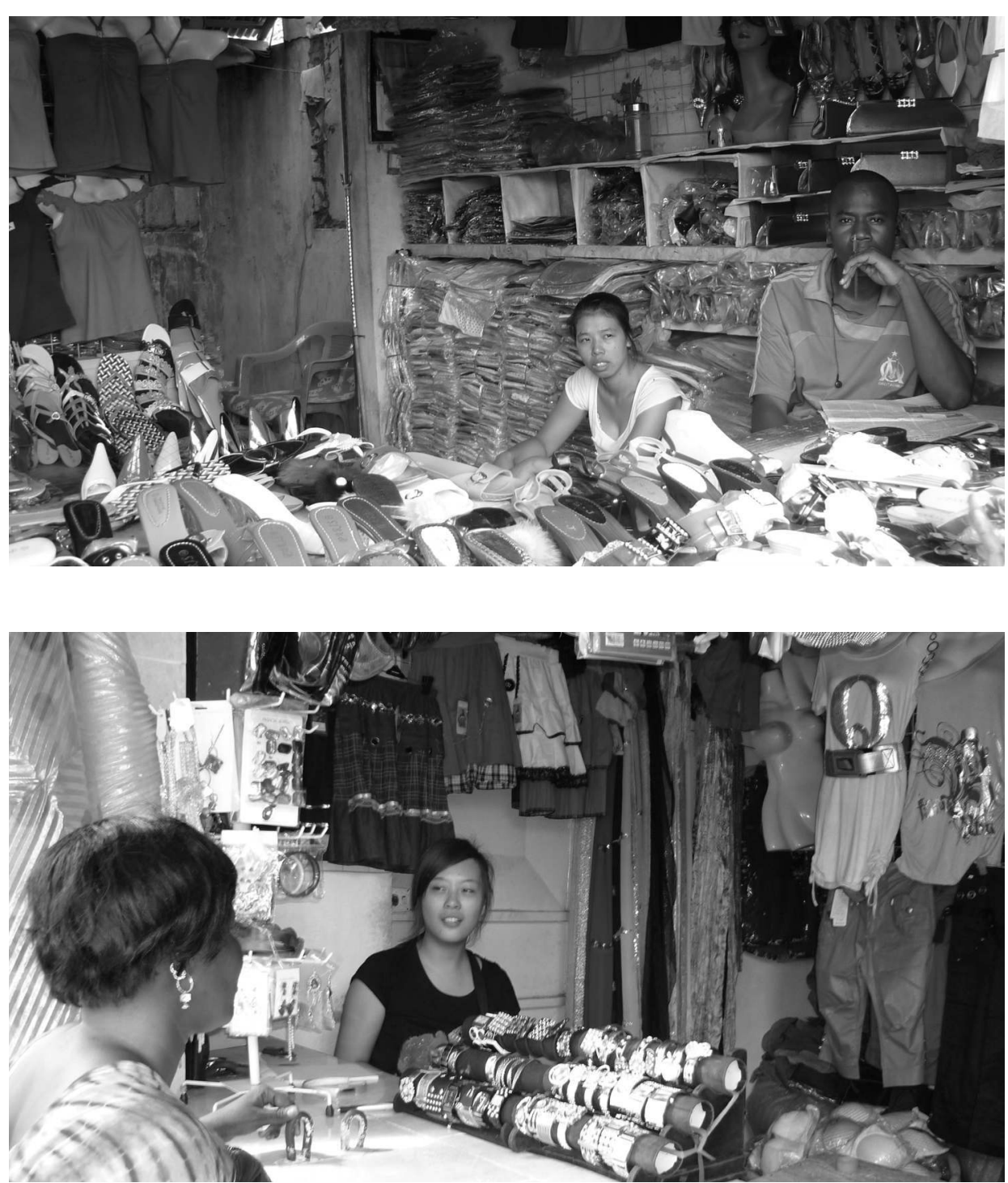

Cantines chinoises (en haut avec un jeune employé). 
à cause du manque de clients. «Un motif qui est incompréhensible» se désole-t-il. "Même pendant la "Tabaski", fête musulmane de l'Aid El Kébir qui commémore le sacrifice d'Abraham, communément appelée fête du mouton, pendant laquelle la plupart des jeunes sénégalais se rendent dans leurs familles situées souvent à l'intérieur du pays, les Chinois donnent à leurs employés uniquement deux jours de repos. Au-delà de ces deux jours, tout employé absent perd son travail». Ce revendeur de produits, installé devant les cantines des nouveaux maîtres du «Centenaire», exemployé des Chinois, nous confie avec tristesse que c'est à cause de son analphabétisme qu'il a été renvoyé par son employeur.

Ces acteurs sénégalais proviennent d'horizons divers. Les marchands ambulants comme les employés viennent pour la plupart de la banlieue (Grand Yoff, Parcelles Assainies, Ouagou Niaye, Thiaroye, etc.), tandis que d'autres habitent le boulevard du Centenaire et ses environs. De plus en plus, ceux qui quittent les zones les plus reculées de la banlieue se rapprochent de leur lieu de travail et trouvent une chambre à la Médina où les loyers sont plus modérés. Cette catégorie est essentiellement composée de jeunes âgés de 20 à 30 ans et de quelques adultes ayant déjà exercé une autre activité avant de s'adonner au commerce. D'origine rurale dans leur majorité, ces marchands sont en quête de travail. Certains ont été manoeuvres mais ont trouvé le commerce avec les Chinois beaucoup plus rentable. D'autres encore ont débuté comme marchands ambulants avant de se stabiliser au niveau du boulevard du Centenaire. Du fait de leur bas prix, les produits chinois sont très accessibles à de nombreux ménages sénégalais confrontés à la baisse de leurs revenus ainsi qu'à l'augmentation du coût de la vie dans la capitale.

Seul un petit nombre parmi les commerçants chinois manie d'autres langues. Quelques mots en français et/ou en wolof permettent de commercer. Dans les boutiques, ils préfèrent qu'un jeune employé sénégalais accueille les clients venus s'approvisionner en marchandises. L'essentiel de leur alimentation est importé de Chine.

Leur commerce dégage une faible fiscalité et l'imitation des produits fabriqués par des artisans locaux (chaussures et sacs en particulier), leur démultiplication en Chine puis leur commercialisation à bas prix à Dakar est en train de «tuer» de manière progressive l'artisanat local. L'arrivée des produits chinois ou de contrefaçon sur le marché national serait responsable de la liquidation(12) de certains artisanats et des problèmes rencontrés dans certains secteurs. Ce processus a été initié par des Chinois mais aussi, il faut le souligner, par certains opérateurs économiques locaux qui se tournent de plus en plus vers la Chine, Dubaï, Singapour et d'autres horizons pour approvisionner le marché local en produits qui concurrencent ceux fabriqués localement.

Le commerce chinois est vivement dénoncé par I'Union Nationale des Commerçants et Industriels du Sénégal (UNACOIS), à cause notamment de ses effets négatifs sur l'économie locale et l'environnement. Les prix pratiqués par les commerçants chinois concurrencent fortement ceux des Sénégalais établis aux marchés Sandaga, Tilène, HLM. «L'inondation du marché par les produits made in China, bien reçue par le consommateur, porte en elle un danger auquel il semble difficile de parer. Comment peuton espérer voir se développer une industrie manufacturière qui serait compétitive quand les produits chinois sont et demeureront longtemps à des prix défiant toute concurrence?» (Richer, 2008)(13). Toutefois, il convient de noter que l'arrivée massive des produits chinois permet à de nombreuses populations africaines dont les revenus sont limités de satisfaire les besoins alimentaires dans la vie des tous les jours.

Au début des années 2000, les commerçants sénégalais ont attiré l'attention de l'opinion sur la mauvaise qualité des produits et une concurrence déloyale en affirmant que les Chinois ne payaient pas les redevances fiscales comme il se doit. 
«lls ne respectent pas la législation douanière et fiscale. Pour payer des taxes réduites, ils déclarent des importations de containers de pièces détachées prêtes à être emboîtées en une demiheure pour donner des produits finis." II n'en demeure pas moins que, malgré ce procès qu'on oppose aux Chinois, leurs produits se vendent en grande quantité au Sénégal, parce que justement ils ont un bon rapport qualité-prix et s'adaptent au marché local.

Les responsables de I'UNACOIS sont montés au créneau pour attirer l'attention des autorités de l'Etat sur les conséquences néfastes de ces activités qui inondent le marché avec des produits de mauvaise qualité. Face à I'UNACOIS, des consuméristes organisés en une entité dénommée Association des Consommateurs Sénégalais (ASCOSEN), appuyés par d'autres segments de la société civile(14), sont venus défendre les commerçants chinois qu'ils disent être en règle avec l'administration. L'ASCOSEN, par la voix de son président, soutenait à l'époque que les pratiques des Chinois n'étaient pas plus illicites que celles des commerçants et marchands sénégalais. II se trouve simplement que les Chinois se sont bien accommodés des artifices connus de leurs hôtes pour minorer leurs charges, à savoir, déclarer une quantité de marchandises dans un container alors qu'il y a parfois près de cent fois plus, déclarer des importations sous forme de pièces détachées, alors qu'il s'agit de produits semi-finis ou purement finis.

Membre du bureau du Groupement économique du Sénégal (GES), première organisation fédérative d'hommes d'affaires du Sénégal indépendant, un haut responsable sénégalais, député de son état, estime que «ces Chinois, non seulement vendent en gros et en détail, mais ils représentent, pour la plupart, des industries de leur pays: ils sont donc industriels, grossistes, demi-grossistes et détaillants, ce qui est inadmissible. Ils gênent ainsi considérablement les nationaux du secteur » et, souligne notre interlocuteur, «dans un pays à très faible production industrielle, cette situation porte préjudice aux nationaux. Car la rapidité dans la méthode et les capacités d'action des Chinois sont insoutenables par leurs homologues sénégalais, voire les autres Africains». A son avis, «il est difficile de faire face à une telle concurrence, parce que plus de $90 \%$ des unités industrielles du Sénégal ne sont pas assez compétitives pour arriver à cette fin».

«Malheureusement, se désole cette personnalité, les règles qui régissent la mondialisation, consolidées, aujourd'hui, par la nature des relations inter-États depuis la normalisation avec la Chine populaire, sont plus favorables aux Chinois». Le rapport de forces est largement favorable au géant asiatique. Conséquence: «les Chinois envahissent le marché de quantités faramineuses de marchandises de basse qualité, à vils prix, qu'ils offrent à des milliers de jeunes. Ces derniers y trouvent leur compte et sont devenus leurs farouches défenseurs». II est urgent de réglementer les activités des Chinois afin de minorer les préjudices causés au plan social, commercial, industriel, etc. Aujourd'hui, même les Moodu-moodu, commerçants sénégalais spécialisés dans l'informel et l'importation des produits asiatiques, ont compris que les Chinois sont venus leur opposer une concurrence avec des méthodes d'une efficacité inégalable et inégalée.

Le député est convaincu qu'il sera difficile à nos gouvernants de faire face à cette situation mieux que les Américains, Européens, Japonais, etc., qui disposent de moyens bien plus importants que ceux des Africains. 


\section{RECONCILIER L'ECONOMIQUE A L'ENVIRONNEMENT: UNE PROBLE- MATIQUE DE GOUVERNANCE ECOLOGIQUE}

II n'y a guère longtemps, le quartier du Boulevard De Gaulle échappait fort bien à bon nombre de tares urbaines. Certes, il est reconnu que le quartier perdait progressivement son charme d'antan à cause du manque d'entretien des espaces verts, mais surtout de la transformation sociale qui affectait les ménages (agrandissement de la taille des ménages, mise en retraite de la génération salariée, pauvreté progressive, etc.). En conséquence, on remarque des difficultés de préservation de l'environnement originel du quartier liées notamment à l'appauvrissement progressif des ménages. Mais la réalité est que les commerces chinois sont implantés dans un quartier, jadis, bourgeois, aujourd'hui entièrement transformé, perdant du coup son aspect résidentiel. En une demidécennie, les Chinois ont démultiplié le rythme de transformation et de dégradation du Centenaire. Ils ont été aidés en cela par les marchands ambulants qui comptent parmi les acteurs importants du système chinois.

Sur le plan environnemental, le commerce chinois a contribué à la dégradation du cadre de vie. L'inscription des Chinois dans l'espace urbain a induit des mutations de la morphologie du boulevard au point de modifier le visage de l'avenue. Avec l'implantation des commerçants chinois suivis des marchands ambulants, cette zone résidentielle a connu progressivement une ambiance de marché à ciel ouvert entraînant une forte image de bidonvilisation progressive du boulevard. L'occupation des devantures des maisons, des allées intérieures, des trottoirs, combinée à la pollution sonore, aux tas d'ordures et à l'encombrement des rues, ont fini par transformer l'image du quartier et fait perdre à cette allée le nom de «plus beau boulevard de Dakar».

Aujourd'hui, une partie des habitants du boulevard migre vers des quartiers plus calmes tandis que d'autres préfèrent rester et s'adapter à la nouvelle situation. Le restaurant Carrefour d'Ivoire était connu pour ses spécialités dans les plats des pays de la sous-région. Sa clientèle était essentiellement composée d'employés des banques, des services et écoles supérieures situés le long du boulevard. En 2006, avec la transformation des Allées du Centenaire suivie de la raréfaction de la clientèle, le propriétaire a préféré quitter le quartier.

Toutefois, de nouvelles structures s'installent. C'est le cas de NECTRANS, une société de transit qui s'est installée aux Allées du Centenaire depuis 6 mois. Même si cette dame transitaire habitant la Médina, interrogée, regrette la bidonvilisation des «Champs Elysées», NECTRANS estime y trouver son compte. II est rejoint dans ses propos par un gérant d'une structure multiservices depuis 2001. De ce point de vue, l'impact négatif du commerce chinois sur les autres activités économiques du boulevard du Centenaire doit être relativisé.

L'organisation spatiale du Boulevard Général de Gaulle est aujourd'hui radicalement différente de l'esprit de l'architecture à la fois sobre et académique qui donnait à la zone son identité de cité des classes émergentes. Quelques édifices tiennent le coup face à cette coulée commerciale venue de la Chine. II s'agit de l'Agence nationale de la Banque centrale des États de l'Afrique de l'ouest (BCEAO), de la Banque de l'habitat du Sénégal (BHS), du Centre africain d'études en gestion (CESAG), de la Radiodiffusion-télévision sénégalaise (RTS), du Building communal. En 1991, seules les allées de promenade des immeubles abritaient les petits commerces de détail mais l'occupation s'était faite de manière ordonnée (Haoua Dia, 1991).

L'évolution de l'activité commerciale sur cette voie est inhérente à certains dysfonctionnements comme les problèmes d'assainissement, de mobilité, la dégradation de l'image du quartier et de la qualité de vie des riverains. Même si les habitants acceptent l'idée de création d'emplois, ils déplorent amèrement la transformation du quartier. L'assainissement du boulevard 
fait défaut. Dès le début de leur installation, il n'y a pas eu de stratégies d'anticipation. Aujourd'hui des mesures correctives ne semblent pas être envisagées. II se pose de plus en plus dans cette zone un véritable problème environnemental à cause de la présence de déchets non biodégradables constitués par les emballages en plastique notamment.

Pour protester contre l'installation des

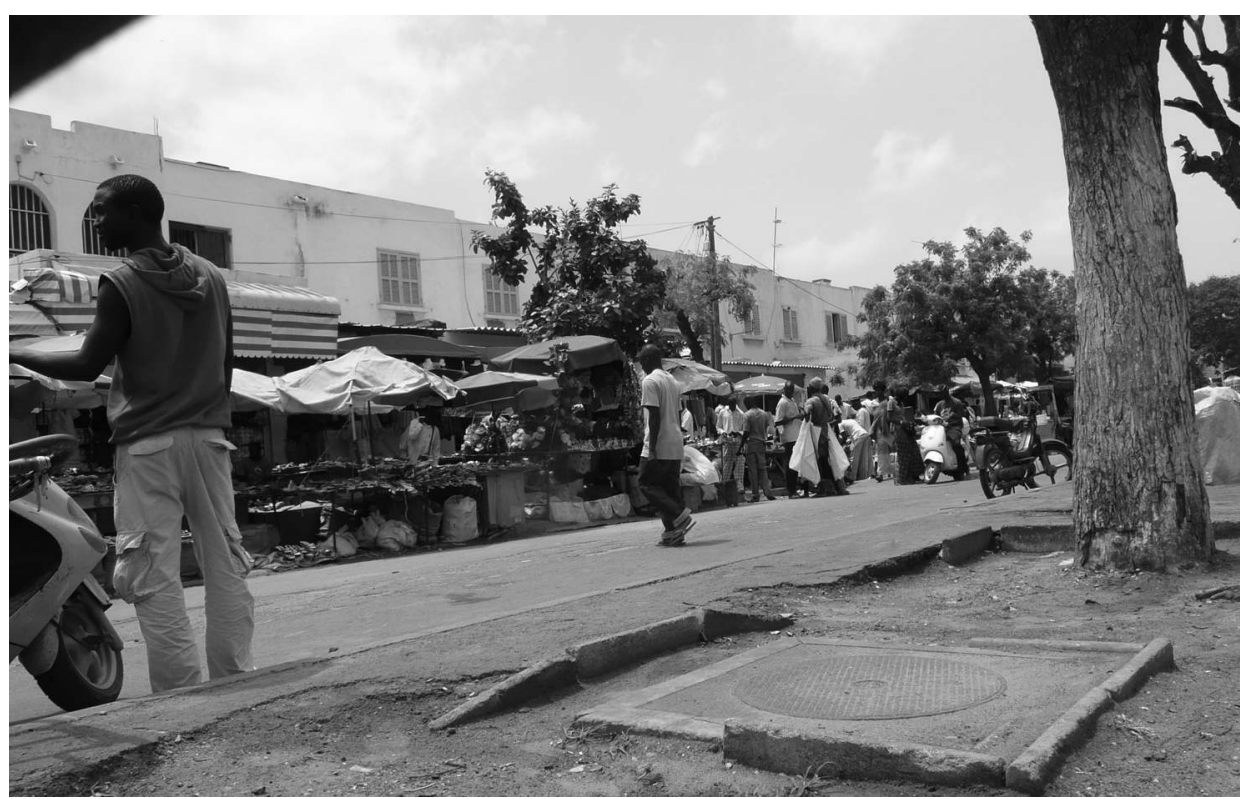

Des étals qui occupent les devantures des cantines.

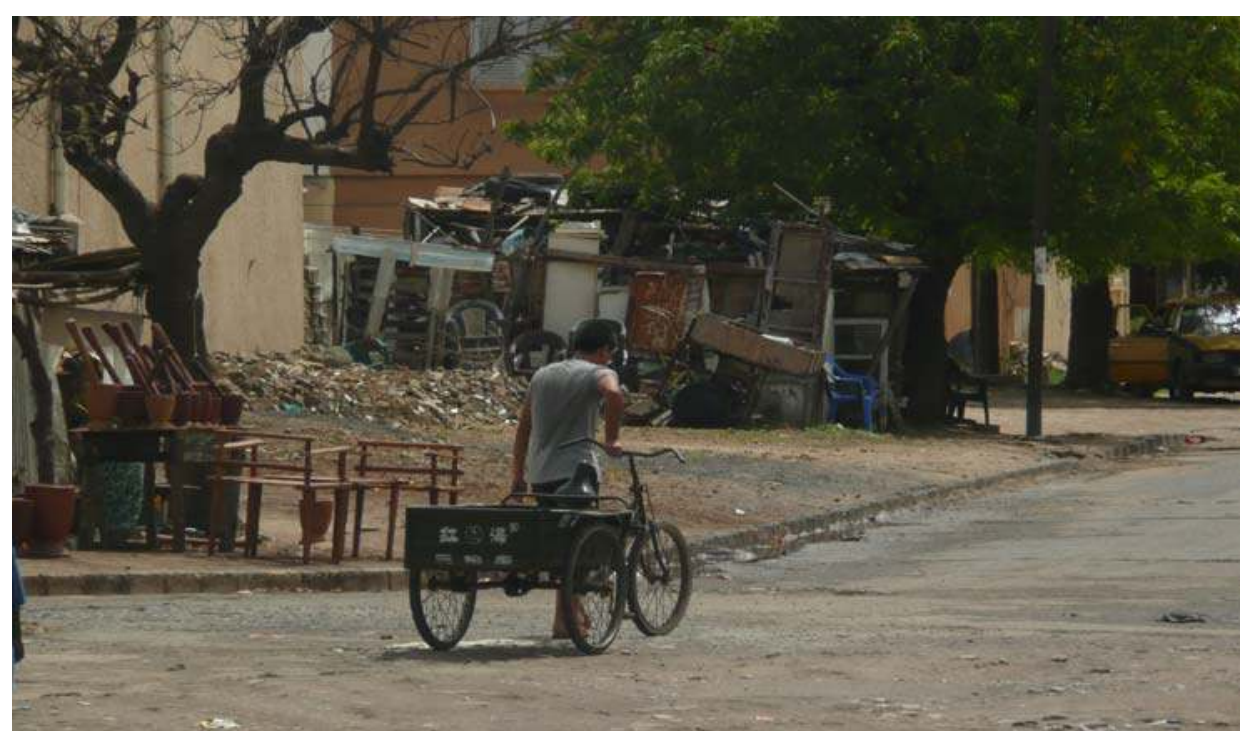

Un tricycle utilitaire (amené pour être réparé) importé par les Chinois pour le transport des marchandises. 
commerces chinois dans ces allées, un habitant a décidé de mettre en place une association des riverains du boulevard de Gaulle en 2006. Selon lui, «le péril chinois à entraîné le «fléau» des marchands ambulants et malgré les multiples rencontres avec le maire de Dakar et le préfet, aucune mesure n'a été prise». Cela lui fait dire que les autorités ont failli à leurs responsabilités de gestion de l'espace urbain. Agé d'une soixantaine d'années, il se souvient avec beaucoup de nostalgie du temps ou il y avait en face des devantures des maisons des espaces verts que les jeunes de son âge entretenaient de manière quasi religieuse. Ce sentiment est partagé par cette dame, employée de banque qui, déjà à l'âge de vingt ans quittait Saint- Louis durant les vacances pour rejoindre des parents au Centenaire. «Je me souviens de la beauté de ce quartier bien entretenu avec ses espaces verts que j'aimais tant. Cela fait longtemps que le boulevard du Centenaire a changé mais cette transformation récente due à la présence des commerçants chinois et des marchands ambulants est une première qui a dénaturé l'image du quartier».

\section{CONCLUSION}

II est impératif de faire une lecture générale de la gestion urbaine dans les pays en voie de développement. L'urbanisation incontrôlée des villes induit un certain nombre de tares liées généralement aux problèmes de pauvreté que les autorités ont toujours du mal à résoudre. Ce phénomène devient surtout insupportable lorsqu'il implique une autre dimension liée à l'environnement: insalubrité, insécurité, pollution, bidonvilisation, inondation, érosion côtière, etc. Si certains de ces problèmes tombent irréversiblement du ciel (les inondations et l'érosion côtière avec certainement une implication anthropique), d'autres viennent d'être majoritairement imputés aux acteurs chinois, exclusivement dans le quartier "Les Allées du Centenaire» de Dakar.

Ces problèmes environnementaux sont toutefois apparus avec un essor de l'économie informelle qui est véritablement une importante source d'emploi pour les jeunes de la banlieue et des régions intérieures. Se confrontent ainsi différentes logiques d'acteurs dont la seule médiation relève d'une réelle volonté de gouvernance, celle capable de concilier l'économique et l'écologique.

L'exemple de la dégradation du boulevard du Général De Gaulle, ex-Allées du Centenaire à Dakar doit amener, en définitive, à poser la question de l'articulation entre la gestion de l'urbain et la préservation d'un environnement sain pour l'ensemble des acteurs. L'occupation anar- chique de cette allée de la métropole dakaroise pose de sérieux problèmes entre les priorités des autorités qui ont en charge les politiques d'aménagement de l'espace urbain et celles des populations. La question de la bonne gouvernance économique dans ses interactions avec la gouvernance écologique transparaît ici comme une question centrale de gestion urbaine de la ville de Dakar. La présence de plus en plus forte des Chinois à Dakar, la concurrence qu'elle induit dans les secteurs de l'artisanat et du commerce (dans ses deux versants import et export), les bouleversements qu'elle entraîne dans l'organisation du secteur informel et du commerce de détail, les conséquences qu'elle engendre dans les attitudes et comportements de jeunes dont beaucoup s'investissent dans le petit commerce des produis chinois, pose avec acuité l'urgence de la prise en charge de cette question. II va de soi qu'entre l'économiquement rentable pour l'Etat et la mairie de Dakar et l'écologiquement viable prôné par les citoyens plus préoccupés par leur cadre et leurs conditions de vie, le choix est vite fait en faveur des Chinois qui apportent une plus-value à l'économie locale sans se soucier de l'environnement des populations.

Aujourd'hui, on peut considérer que les Chinois sont partie prenante dans le jeu des acteurs dans l'agglomération de Dakar car, au-delà de leur zone d'implantation, ils ont opéré un maillage territorial 
qui permet à leurs produits de se retrouver partout au Sénégal et dans la sousrégion, sans qu'ils aient besoin de se déplacer.

II faut cependant reconnaître que l'irruption des Chinois dans l'espace national a fortement limité la fraude en provenance de la Gambie en ce qui concerne les produits vestimentaires, mais a également ébranlé les fondements de l'artisanat local dont l'élan s'est brisé du fait de cette concurrence.

Quelle réponse faut-il apporter à une telle situation? Comment assurer les équilibres qui ont fondé l'économie sénégalaise avec, d'une part, un secteur informel dynamique et générateur d'emplois et servant de soupape de sécurité face à la crise des emplois dans l'administration et le secteur formel, l'émergence de cette nouvelle catégorie d'acteurs que sont les Chinois et, d'autre part, la nécessité de moderniser l'agglomération dakaroise, d'améliorer le cadre et les conditions de vie des populations? Voilà le véritable enjeu qui interpelle les pouvoirs publics qui doivent davantage opter pour une approche participative et inclusive des populations afin de déterminer les orientations à mettre en oeuvre dans l'optique d'une bonne gouvernance métropolitaine, dans l'intérêt, bien entendu, des différents acteurs.

\section{BIBLIOGRAPHIE}

- BREDELOUP S. \& BERTONCELLO B. (2006), «La Migration chinoise en Afrique: accélérateur du développement ou sanglot de l'homme noir?", Afrique Contemporaine, 218, pp. 199-224.

- CARROUE L. (2005), Géographie de la mondialisation, Armand Colin, Paris, $256 \mathrm{p}$.

- CHAUDOIR Ph. (2002), "L'émergence comme paradigme du renouvellement des politiques culturelles publiques, le cas des arts de la rue», L'observatoire, 22 janvier 2002

- DÉFIS SUD (2007), Dossier No 79 sur la politique de la Chine envers le continent noir, septembre octobre 2007, bimestriel, publication de SOS-FAIM, Rue aux Laines, 4, 1000 Bruxelles, Belgique.

- DIA H. (1990-1991), Occupation et utilisation de l'espace dans le secteur du Boulevard Général de Gaulle: de la place Sfax à la place de la Nation, mémoire de maîtrise, Université de Dakar, 99 p.

- DIOP A. (2005), Villes et Aménagement du Territoire au Sénégal, Thèse de doctorat d'Etat, UCAD, $405 \mathrm{p}$.

- DIOP A. (2008), Enjeux urbains et développement territorial en Afrique contemporaine, Karthala, $176 \mathrm{p}$.

- DIOP A. (dir.) (2008), Développement local, gouvernance territoriale, Enjeux et perspectives, Karthala, $285 \mathrm{p}$.

- DIOP MAGUETTE S. (1989-1990), Occupation et utilisation des espaces urbains, Approches socio-spatiales de l'encombrement des rues de Dakar. Cas du quartier Plateau, mémoire de maîtrise, Université de Dakar, 148 p. .

- DIOUF M. (2000), “Commerce et cosmopolitisme, le cas des diasporas mourides du Sénégal» Bulletin du Codesria, 1. - KERNEN A. (2004), La Chine vers l'économie de marché: les privatisations à Shenyang, Paris, Karthala.

- LAFARGUE F. (2005), «La Chine, une puissance africaine», Perspectives chinoises, 90, juillet-août 2005, pp. 19-30.

- MICHEL S. \& BEURET M. (2008), La Chinafrique, Grasset et Fasquelle, 348 p.

- MIOSSEC J.M. (2002), «De la géographie prioritaire à l'unité de la cité: gérer le changement urbain", Bulletin de la Société Languedocienne de Géographie Espace et Territoires, 33, janvierdécembre 1999, pp. 235-258.

- NDIAYE M. (1998), L'éthique ceddo et la société d'accaparement ou les conduites culturelles des Sénégalais d'aujourd'hui, Tome 1, Le Gorgui, PUD, 418 p.

- NGOM MAME Ch. (2001-2002), Les marchés, enjeux politiques et économiques dans la gestion communale: Cas de Pikine, mémoire de maîtrise, Université Cheikh Anta Diop, 84 p.

- OSMONT A. (1995), La banque mondiale et les villes, du développement à l'ajustement, Paris, Karthala, $309 \mathrm{p}$.

- RICHER Ph. (2008), L'offensive chinoise en Afrique, Karthala. 
- ROCCA J.L. (2006), La condition chinoise: capitalisme, mise au travail et résistances dans la Chine des réformes, Paris, Karthala

- SANTOS M. (1975), L'espace partagé: les deux circuits de l'économie urbaine des pays sous développés, Paris, Genin, Librairies techniques, $405 \mathrm{p}$.

- SECK A. (1970), Dakar métropole ouest africaine, IFAN-Dakar, 516 p.
(1) Cette politique était axée sur quatre secteurs clés: l'agriculture, l'industrie, la science et la technologie ainsi que la défense nationale. La modernisation de ces quatre secteurs avait pour objectifs de rendre la Chine prospère et d'accroître son influence sur le plan international.

(2) Les investissements étrangers (IDE) en Chine sont passés de 250 milliards de dollars en 1998 à plus de 550 milliards en 2005. D'ailleurs, la Chine reçoit plus de la moitié des investissements étrangers réalisés en Asie.

(3) L'un de ses temps forts a été, d'une part, la publication, le 12 janvier 2006, de la «Politique de la Chine à l'égard de l'Afrique» (www.fmprc.gov.cn/fra/zxxx/ t230916.htm); lire aussi le discours du président Hu Jintao dans http://english. focacsummit.org/2006-11/04/content. 4978.htm, et d'autre part le Forum entre l'Afrique et la Chine de novembre 2006.

(4) A ce propos, Serge Michel et Michel Beuret soulignent que le visa sénégalais est l'un des plus difficiles à obtenir pour les migrants chinois et donc le plus cher sur le marché noir. C'est pourquoi certains d'entre eux transitent généralement par d'autres pays africains comme la Guinée avant d'entrer clandestinement au Sénégal.

(5) Le Moodu-moodu ou Baol Baol est un migrant ayant une origine profondément rurale. Diouf Mamadou (2000) dit qu'il s'agit d'une catégorie sociale qui se "construit dans une errance, menant une vie de sacrifice, pleine de privations". Le Moodu-moodu, c'est aussi toute une histoire, celle d'un migrant, un «nomade» sur le chemin de la fortune. Le souci du gain acquis à la sueur de son front lui fait quitter son village pour une capitale régionale, ensuite la capitale nationale Dakar et parfois le monde, en particulier, les EtatsUnis et l'Italie qui renferment les plus grandes colonies de Moodu-moodu. Ndiaye Malick (1998), note que «le Moodu-moodu... est de plus en plus un mouvement d'affirmation socioculturelle et économique des ruraux du bassin arachidier, fruit de l'exode de la fin des années 60 et durant la décennie 70-80, dans le commerce et les affaires urbaines. Aujourd'hui, il y a un glissement sémantique progressif qui assimile le Moodumoodu à l'émigré parti chercher fortune en Europe ou en Amérique».

(6) Par sa position privilégiée au niveau du centre-ville, le marché Sandaga demeure un pôle commercial très dynamique. C'est l'actuel plus grand marché de Dakar qui "de marché de denrées alimentaires et d'étoffes est devenu un centre de vente d'articles électroniques venant d'Asie (Hong Kong), du Moyen Orient (Djeddah) et d'Amérique (New York)» (Ebin, 1992, cité par Diouf (2000), "Commerce et cosmopolitisme, le cas des diasporas mourides du Sénégal», Bulletin du Codesria, $\left.n^{\circ} 1\right)$.

(7) La rénovation d'une partie de la Médina, quartier indigène créé en 1914 par les colonisateurs français, a donné naissance au quartier dénommé Allées du Centenaire. Ce quartier fut érigé en 1967, en lieu et place des quartiers de bidonvilles de Thieurigne et Fith Mith. II est organisé de part et d'autre de l'artère baptisée tour à tour Boulevard de la liberté, Allées du Centenaire et enfin Boulevard du Général de Gaulle.

(8) Les quartiers des HLM furent réalisés par l'Office des Habitations à Loyer Modéré, créé en 1960. Sa principale raison d'être était la construction de logements pour les économiquement faibles, incapables d'accéder aux logements offerts par la SICAP.

(9) II s'agit des quartiers dont les logements furent réalisés par La Société Immobilière du Cap Vert qui a été créée en 1951. Ces logements économiques sont censés répondre aux possibilités financières de la population, mais pour ces deux sociétés, les objectifs sociaux poursuivis sont loin d'être aujourd'hui une réalité. 
(10) Occupant une position centrale, le boulevard est entouré par les plus importants marchés Sandaga, Colobane, HLM, Tilène.

(11) II ressort de nos entretiens avec les populations riveraines des Allées du Centenaire que la très forte demande en établissements de commerce par les Chinois a été à l'origine de la construction rapide et massive de cantines qui, visiblement, ne répondent à aucune norme d'urbanisme. Les cantines désignent ici des établissements commerciaux allant d'un standing global sommaire à moyen. Le phénomène de "cantinisation» constitue un des faits marquants du paysage urbain sénégalais. Concomitamment observée avec l'essor de l'économie informelle, et plus particulièrement en rapport aux initiatives individuelles des populations, l'ouverture de cantines dans les pourtours des rues de Dakar est vertigineuse. II s'agit dans la plupart des cas de nouvelles constructions ou de modifications issues d'une maison familiale dont le propriétaire envisage une location qui fait gagner des revenus supplémentaires. Depuis quelques années, les collectivités locales en sont parmi les initiateurs. Pour des soucis d'aménagement, elles en construisent massivement au niveau des marchés afin d'éviter l'occupation des rues.

(12) II s'agit d'une accélération d'un processus que Jean Roch avait bien observé: la logique adverse entre certains artisanats utilitaires (employant en partie des migrants saisonniers) et les grandes entreprises, comme entre la société BATA et les artisans de la chaussure et des tanneries, les forgerons et la SISCOMA, etc. Cf. Roch Jean (1976), "La richesse paysanne en bassin arachidier sénégalais: inventaires et essai d'évaluation», Cah. ORSTOM, sér. Sci. Hum., XIII, 4, pp. 383407.

(13) La concurrence chinoise est destructrice. Dans de nombreux pays africains, le commerce chinois a eu de graves conséquences sur l'économie locale: fermeture d'une douzaine d'industries en Afrique du Sud, fermeture d'une usine textile en Zambie, arrêt de l'industrie textile qui était la seule au Lesotho, entre autres.

(14) Michel et Beuret soulignent que l'arrivée des Chinois a involontairement structuré la société civile sénégalaise. Dans sa campagne de défense des commerçants chinois contre ce qu'elle appelle «l'intolérance, le racisme et la xénophobie de I'UNACOIS", I'ASCOSEN a été soutenue par des mouvements de défense des droits et des syndicats de travailleurs.

\author{
Amadou Diop \\ Professeur \\ Université Cheikh Anta Diop de Dakar \\ gerad@orange.sn
}

manuscrit déposé en décembre 2008; révisé en avril 2010 